\title{
ACCELERATOR PHYSICS
}

\section{OVERCOMING INTRINSIC AND SYNCHROTRON DEPOLARIZING RESONANCES WITH A SIBERIAN SNAKE}

\author{
J.E. Goodwin, H-O. Meyer, M.G. Minty, P.V. Pancella, R.E. Pollock, T. Rinckel, \\ M.A. Ross, F. Sperisen, E.J. Stephenson, and B. von Przewoski \\ Indiana University Cyclotron Facility, Bloomington, Indiana 47405 \\ E.D. Courant, S.Y. Lee and L.G. Ratner \\ Brookhaven National Laboratory, Upton, New York 11973
A.D. Krisch, R.S. Raymond, T. Roser, J.A. Stewart and B. Vuaridel Randall Laboratory of Physics, \\ University of Michigan, Ann Arbor, Michigan 48109
}

We overcame the strong $\mathrm{G} \gamma=-3+\nu_{\mathrm{y}}$ intrinsic depolarizing resonance using a Siberian Snake acting on a stored beam of $177 \mathrm{MeV}$ polarized protons at the Indiana University Cooler Ring. We also saw the first evidence for a synchrotron depolarizing resonance in a proton ring. Two synchrotron resonances were studied by varying the of accelerating voltage and the imperfection field at $104 \mathrm{MeV}$. The Siberian Snake also overcame these synchrotron depolarizing resonances. The main element of our Siberian Snake was a superconducting solenoid magnet.

To demonstrate with good precision that the $\mathrm{G} \gamma=2$ intrinsic depolarizing resonance studied earlier has a strong effect at $104 \mathrm{MeV}$, we turned the Snake off and injected protons polarized in the vertical direction. We then studied the depolarizing resonance with a high sensitivity. As shown in Fig. 1 we again saw the fairly sharp peak reported earlier; however, the imperfection field in the Cooler solenoids was now varied in much finer steps than before. This fine detail gave a most surprising result; there is an extremely sharp dip on each side of the peak. The polarization appears to go to zero in each dip. These narrow dips were unexpected; they seemed to be some new type of depolarizing resonance. These dips were at first difficult to believe because they were so narrow, but they were reproducible. Moreover, as shown in Fig. 1, these narrow dips occur simultaneously in the radial polarization at exactly the same $\int \mathrm{B} \cdot \mathrm{d} \ell$ values.

Of the several ideas about these narrow dips, the most likely explanation seemed to be synchrotron depolarizing resonances. For the Cooler Ring at $104 \mathrm{MeV}$ the synchrotron tune is related to the rf accelerating voltage, $V_{\mathrm{rf}}$ in Volts, by the equation

$$
\nu_{\mathrm{syn}}=7.510^{-5}\left(\mathrm{~V}_{\mathrm{rf}}\right)^{1 / 2}
$$

We tested the synchrotron depolarizing resonance hypothesis by varying the rf accelerating voltage in the Cooler Ring to study the effect of $V_{x f}$ on the polarization. We set the longitudinal solenoid imperfection field in the center of the left dip and then measured 


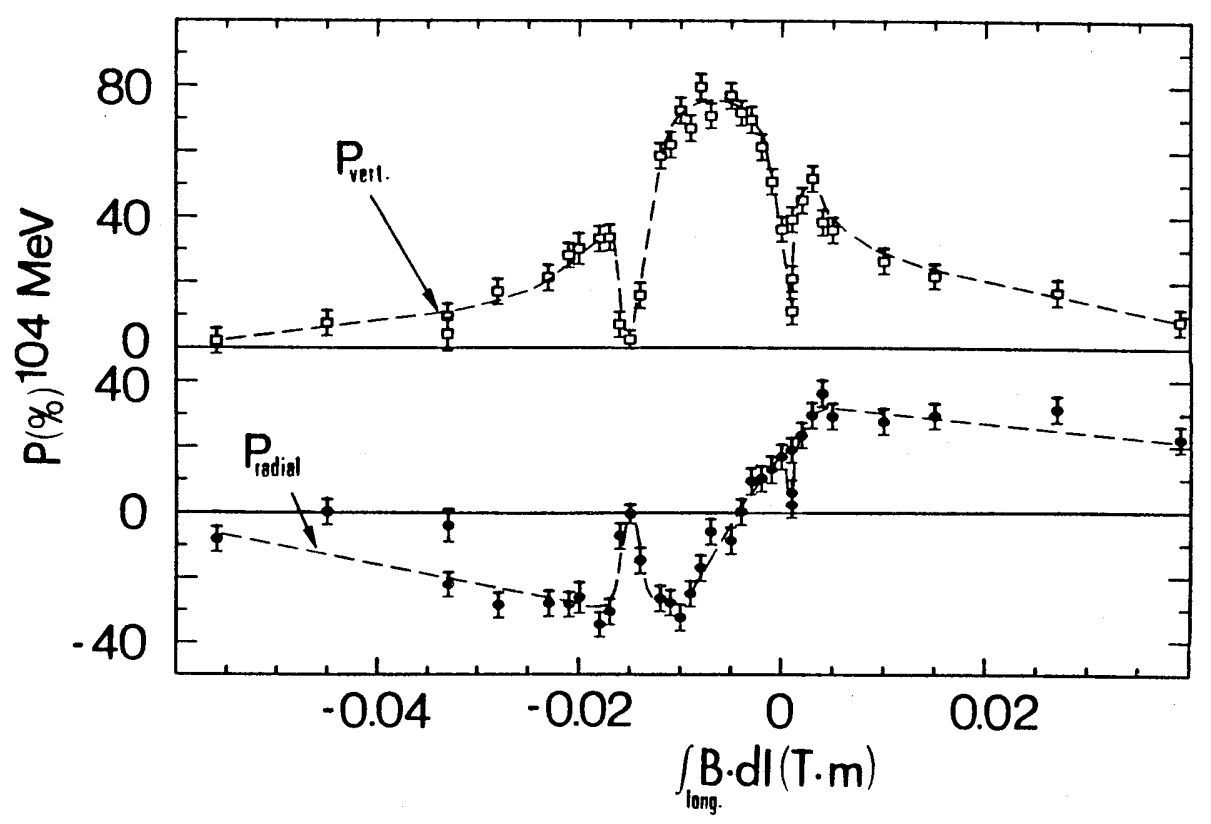

Figure 1. The vertical (squares above) and radial (circles below) components of the beam polarization at $104 \mathrm{MeV}$ are plotted against the longitudinal magnetic field integral in the Cooler solenoids with the Snake off and the injection of vertically polarized protons. There is a systematic normalization uncertainty of about $\pm 4 \%$. The dashed curves are hand-drawn lines to guide the eye.

the vertical polarization while varying $\mathrm{V}_{\mathbf{r f}}$. This data, which is shown in Fig. 2, clearly demonstrates that the depolarization is related to $V_{\mathrm{rf}}$. This appears to be direct evidence that the dips are indeed synchrotron depolarizing resonances.

We then tested the ability of a Siberian Snake to overcome these synchrotron depolarizing resonances. With the imperfection solenoid field still set at the center of the left dip, we turned on the Snake and then measured the radial polarization in the ring while varying the rf voltage. As shown in Fig. 2, the Snake eliminated all observable effects of the synchrotron depolarizing resonance, giving a further indication of a Siberian Snake's ability to overcome all types of depolarizing resonances.

We also made the first test of a Siberian Snake's ability to overcome an intrinsic depolarizing resonance. An intrinsic resonance is caused by the vertical betatron oscillations which are due to the focusing horizontal quadrupole fields in a proton ring. An intrinsic depolarizing resonance occurs whenever the spin precession frequency becomes equal to the frequency of encountering these horizontal fields. We studied the strong $\mathrm{G} \gamma=-3+\nu_{\mathrm{y}}$ intrinsic depolarizing resonance by injecting and storing polarized protons at $177 \mathrm{MeV}$; we then measured the polarization while using the Cooler Ring quadrupoles to vary the vertical betatron tune, $\nu_{y}$, to different values near the resonance. We first injected vertically polarized protons with the Snake off and measured the vertical polarization. We then injected horizontally polarized protons with the Snake on and measured the radial polarization as seen in Fig. 3. Clearly with the Snake off the beam was totally depolarized for $\nu_{y}$ values between about 5.125 and 5.140 . With the Snake turned on we maintained full polar- 
Figure 2. The beam polarization in each stable polarization direction at $104 \mathrm{MeV}$ is plotted against the voltage of the radio frequency accelerating cavity in the Cooler Ring. The large circles are the radial polarization with the Snake on and the injection of horizontally polarized protons. The small squares are the vertical polarization with the Snake off and the injection of vertically polarized protons. The maximum vertical polarization is reduced significantly by a mismatch of the stable spin directions at injection. There is a systematic normalization uncertainty of about $\pm 4 \%$. The dashed curves are hand-drawn lines to guide the eye.

Figure 3. The beam polarization in each stable spin direction at $177 \mathrm{MeV}$ is plotted against the vertical betatron tune $\nu_{y}$. The large circles are the radial polarization with the Snake on and the injection of horizontally polarized protons. The small squares are the vertical polarization with the Snake off and the injection of vertically polarized protons. There is a systematic normalization uncertainty of $\pm 4 \%$. The dashed curves are hand-drawn lines to guide the eye.
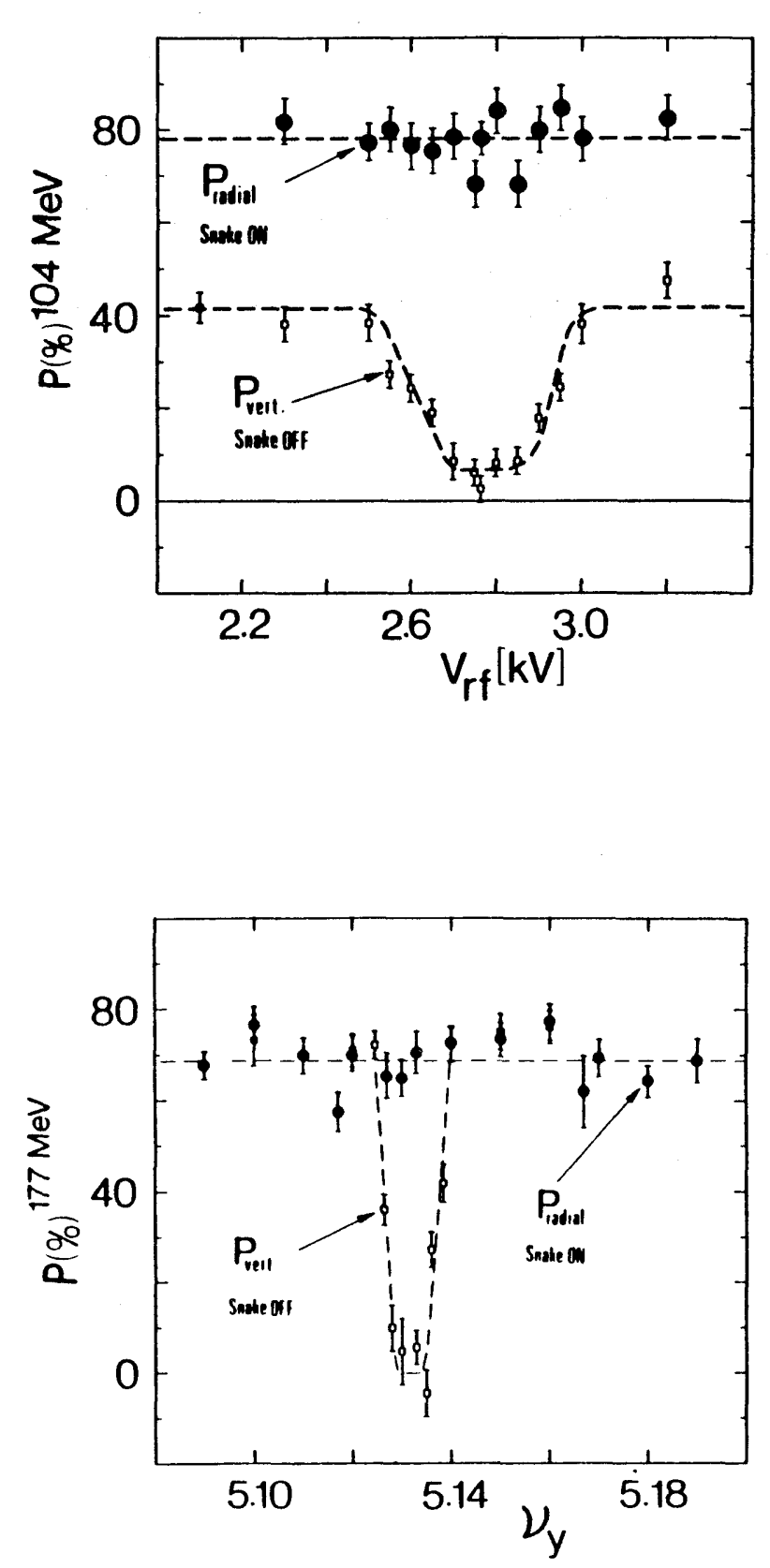

ization for all measured tune values. These data are a direct demonstration of the ability of a Siberian Snake to overcome a strong intrinsic depolarizing resonance. This data also gave an absolute calibration of the energy $m \gamma$ of the Cooler Ring to a precision of about $\pm 0.03 \%$.

We are now becoming more optimistic that Snakes can be used to overcome all types of depolarizing resonances. Thus it may now be technically feasible to accelerate polarized beams to very high energies at facilities like $\mathrm{KAON}, \mathrm{U}-70, \mathrm{RHIC}$, the Fermilab main ring, the Tevatron, HERA, UNK, and the SSC. 
We would like to thank S.R. Mane and K.M. Terwilliger for their help with the earlier part of the experiment. We are grateful to T. Bertuccio, J.M. Cameron, G. East, T. Ellison, D.L. Friesel, J. Hicks, K. Komisarcik, R. Palmer, W.T. Sloan, P. Schwandt and the entire IUCF staff for the successful operation of the IUCF Cyclotron and Cooler Ring and the Siberian Snake. We are grateful to O. Chamberlain, W. Fry, J. Geller, R. Raylman, H. Sato, L.C. Teng, and U. Wienands for advice and help. This research was supported by grants from the U.S. Department of Energy and the U.S. National Science Foundation. 